\title{
COMENTARIO DE EXPERTO
}

\section{Dr Pablo Brockmann}

En el presente número de Neumología Pediátrica se abordan distintos aspectos de los tratornos respiratorios del sueño (TRS) en niños. Por un lado, un artículo resume la epidemiología de esta verdadera "epidemia" diagnóstica, con especial énfasis en las cifras chilenas, las cuales son alarmantes por la alta prevalencia de los TRS.

En otro articulo, se aborda el impacto que tienen los TRS en niños con necesidades especiales, niños con Sindrome de Down. En estos grupo, el diagnóstico debe ser cada vez más precoz y no se debe escatimar en la derivación precoz para realizar exámenes diagnósticos.

El enfrentamiento diagnóstico justamente es un desafio, que pone en énfasis la necesidad de buscar alternativas, dada la escasez de recursos como la Polisomnografía. Este fenómeno, que no solo afecta a Chile, si no toda la región y se ha descrito en Europa y Estados Unidos también, implica largas listas de espera, costos elevados y un diagnóstico muy dependiente de profesionales muy capacitados en sueño, escasos a nivel nacional e internacional. Es por esto que estudios abreviados como la poligrafía han captado la atención de los clínicos y médicos que se dedican a sueño en niños ${ }^{(1)}$. La poligrafía ha demostrado en varios trabajos originales como el del grupo español de Alonso Alvarez $z^{(1,2)}, 0$ el grupo de Chicago ${ }^{(3)}$, tener un rendimiento diagnóstico excelente para el diagnóstico de apneas obstructivas del sueño en niños. Nuestro grupo ha realizado la validación de este procedimiento en niños ${ }^{(4)}$ y en niños con síndrome de Down ${ }^{(5)}$. Actualmente hay cada vez mas centros en Chile que han implementado un estudio poligráfico cardiorespiratorio tanto domiciliario como hospitalario para el enfrentamiento de esta gran tasa de TRS que se ha registrado.

En otro de los articulos presentados en el presente número de la revista, se aborda la utilidad de los cuestionarios para el diagnóstico de Ios TRS. En este punto, ha habido gran interés en el desarrollo de herramientas simples, eficaces y de bajo costo para realizar screening en grandes poblaciones. Uno de los cuestionarios más utilizados y que ha demostrado validez en nuestro medio es el Pediatric Sleep Questionnaire (PSQ), que ha logrado diagnosticar sobre el $90 \%$ de los niños referidos a nuestro centro por apnea obstructiva del sueño utilizando ciertos puntos de corte específicos. ${ }^{(6)}$

Se hace referencia al impacto que tienen los TRS en niños con enfermedades neuromusculares, al igual que en niños con Sindrome de Down, en este grupo el diagnóstico precoz se hace aún más perentorio. Por otra parte, el enfoque terapeutico se basa habitualmente en el inicio de ventilacion no invasiva, la cual implica un largo camino de seguimiento y titulacion con poligrafia o polisomnografia. En este grupo, la derivación oportuna y el trabajo multipdisciplinario hacen una gran diferencia en pronóstico y morbilidad a largo plazo.

Por último, cabe mencionar el avance en las técnicas de biología molecular y genética para el diagnóstico no tan solo de los TRS per se, sino también para la identificación de aquellos niños(as) con riesgo para desarrollar alteraciones neurocognitivas. En uno de estos estudios Kheirandish-Gozal y cols demostraron que una serie de proteínas urinarias eran marcadores de daño neurocognitivo en niños con TRS ${ }^{(7)}$. Por otro lado, marcadores inflamatorios como monocitos han demostrado diferenciar niños con y sin TRS ${ }^{(8)}$. La epigenética, es decir los cambios en metilación postnatales en ciertos genes han demostrado ser un nicho de especial interés para el desarrollo de nuevas técnicas de pesquisa de TRS. ${ }^{(9)}$

En resumen, el diagnóstico de los TRS abre un espectro enorme de posibilidades que han aumentado en los últimos años. El buen y criterioso uso de las herramientas diagnosticas permitirá en el futuro un diagnóstico más precoz y certero de aquellos niños con TRS y real riesgo de presentar consecuencias metabólicas y neurocognitivas.

\section{REFERENCIAS}

1. Alonso-Alvarez M T-SJ, Cordera-Guevara J, Navazo-Eguia A, Ordax-Carbajo E. Respiratory polygraphy for diagnosis and evolution of sleep apnea/hypopnea syndrome in children. Eur Respir Soc. 2008.

2. Alonso-Alvarez ML, Teran-Santos J, Ordax Carbajo E, Cordero-Guevara JA, Navazo-Eguia Al, Kheirandish-Gozal L, et al. Reliability of home respiratory polygraphy for the diagnosis of sleep apnea in children. Chest 2015; 147:1020-8.

3. Tan HL, Kheirandish-Gozal L, Gozal D. Pediatric Home Sleep Apnea Testing: Slowly Getting There! Chest 2015;148:1382-95.

4. Brockmann PE, Perez JL, Moya A. Feasibility of unattended home polysomnography in children with sleep-disordered breathing. Int J Pediatr Otorhinolaryngol. 2013;77:1960-4.

5. Brockmann PE, Damiani F, Nunez F, Moya A, Pincheira E, Paul MA, et al. Sleep-disordered breathing in children with Down syndrome: Usefulness of home polysomnography. Int J Pediatr Otorhinolaryngol. 2016;83:47-50. 6. Bertran K, Mesa T, Rosso K, Krakowiak MJ, Pincheira E, Brockmann PE. Diagnostic accuracy of the Spanish version of the Pediatric Sleep Questionnaire for screening of obstructive sleep apnea in habitually snoring children. Sleep Med. 2015;16:631-6.

7. Kheirandish-Gozal L, McManus CJ, Kellermann GH, Samiei A, Gozal D. Urinary Neurotransmitters Are Selectively Altered in Children with Obstructive Sleep Apnea and Predict Cognitive Morbidity. Chest 2013;143:1576-83. 8. Kheirandish-Gozal L, Wang Y, Duggan RC, Harshan Vardhan S, Tan HL, Molero Ramirez $\mathrm{H}$, et al. Nitric oxide production by monocytes in children with OSA and endothelial dysfunction. Clin Sci (Lond). 2014;127:323-30.

9. Kheirandish-Gozal L, Khalyfa A, Gozal D, Bhattacharjee R, Wang Y. Endothelial Dysfunction In Children With Obstructive Sleep Apnea Is Associated With Epigenetic Changes in the eNOS Gene. Chest 2013;143:971-7 